13

\title{
Атомно-слоевое осаждение тонких пленок на $3 D$-наноструктуры: влияние наклона стенок и аспектного отношения тренчей
}

\author{
(C) А.В. Фадеев, К.В. Руденко \\ Физико-технологический институт РАН, \\ 117218 Москва, Россия \\ e-mail: AlexVFadeev@gmail.com
}

(Поступило в Редакцию 10 ноября 2017 г.)

Развита теоретическая модель, предсказывающая пространственный профиль пленки, выращиваемой на стенках методом атомно-слоевого осаждения. Модель учитывает возможность исходного отклонения стенок тренча от вертикали, а также динамическое изменение аспектного отношения структуры по мере роста пленки в наноразмерных тренчах. Теоретически исследована зависимость результирующей толщины и конформности пленки от параметров процесса атомно-слоевого осаждения.

DOI: $10.21883 / J T F .2018 .10 .46504 .2550$

\section{Введение}

В настоящее время в наноэлектронике широко применяется метод атомно-слоевого осаждения (ALD), одним из основных преимуществ которого является высокая конформность процесса осаждения на трехмерных структурах со сложной геометрией [1]. Конформность обеспечивается изотропностью процесса хемосорбции первого прекурсора, а прецизионный контроль толщины растущей пленки - самонасыщением реакции первого и второго прекурсоров в каждом ALD-цикле. В нулевом приближении полагается, что в идеальном процессе $\mathrm{ALD}$ за один цикл вырастает один монослой пленки конформно, т.е. с равной толщиной, покрывающей любую геометрически сложную поверхность.

Однако при выращивании тонких пленок методом ALD на $3 D$-структурах с высоким аспектным отношением (отношение глубины $H$ к ширине $W$ микроструктуры), например, в канавках (тренчах) приборных структур наноэлектроники, лимитирующей стадией, влияющей на процесс в целом, может оказаться транспорт паров/газов прекурсоров к труднодоступной поверхности. Поэтому определение оптимальных условий ALD при различных аспектных отношениях 3D-структур, включая дозирование, длительности и температуры ALD-процесса - предмет активных теоретических и экспериментальных исследований [1-7]. Подавляющее большинство теоретических работ представлено численным моделированием при помощи метода Монте-Карло, например [3-5]. При этом верификация получаемых при моделировании зависимостей степени покрытия стенок $3 D$-структур осуществляется сравнением с экспериментальными зависимостями толщины выращенной пленки $h(z)$, нормированной затем на количество ALD-циклов. Указанный подход не учитывает влияния возможной неконформности осаждения пленки по мере ее роста в каждом ALD-цикле и динамического изменения геометрии тренча в процессе осаждения, когда толщина осаждаемой пленки и характерный размер $3 D$-структуры сопоставимы.

Целью настоящей работы является теоретическое исследование того, как исходный наклон стенок тренча и его возможное изменение по мере роста пленки, а также динамически меняющееся аспектное отношение микроструктур влияют на результирующие параметры конформности пленок, осаждаемых на поверхность тренчей методом ALD.

\section{1. Система кинетических уравнений, описывающих рост пленки}

Ранее [8] была предложена кинетическая модель, которая позволяет рассчитать пространственный профиль пленки, выращенной на стенках высокоаспектных вертикальных тренчей. Модель позволяет определить минимальное время подачи прекурсора, необходимое для конформного покрытия стенок тренча. Предложенная модель содержала следующие допущения:

- параметры газа, подаваемого в камеру реактора, а также размеры тренчей, соответствуют молекулярному режиму течения газа внутри тренча;

- концентрация молекул прекурсора в газовой фазе над плоской поверхностью постоянна, а их распределение по скоростям подчиняется распределению Максвелла;

- при взаимодействии с поверхностью частица может либо прилипнуть к поверхности с вероятностью

$$
p=s(1-\Theta) \text {, }
$$

где $\Theta$ - степень покрытия стенок тренча, а $s$ - коэффициент прилипания молекул прекурсора, либо отразиться с вероятностью 1-р по закону косинуса;

- отраженная частица приобретает температуру поверхности;

- пренебрегается возможностью поверхностной диффузии адсорбированных частиц. 
С учетом сделанных предположений кинетическое уравнение, описывающее зависимость степени покрытия стенок тренча от времени подачи прекурсора, имеет вид

$$
n_{0} \frac{d \Theta}{d t}=\left(J_{e}+J_{r}\right) s(1-\Theta)
$$

где $n_{0}$ - поверхностная концентрация прекурсора в адсорбированном монослое, а $J_{e}$ и $J_{r}-$ плотности потоков частиц на площадку $d S$ тренча из газовой фазы и за счет отражений от других частей тренча соответственно.

Аналогично [8] будем исследовать рост пленки $\mathrm{HfO}_{2}$ ALD-методом, использующим гетерогенную реакцию тетраэтилметиламиногафния (ТЕМАН) и паров воды. B ALD-цикле плотность потока молекул TEMAH на несколько порядков меньше плотности потока молекул воды. Это обусловлено большей молекулярной массой и низким давлением насыщенных паров ТЕМАН по сравнению с парами воды. В результате время ALD-цикла в первую очередь лимитируется временем подачи первого прекурсора (ТЕМАН), поэтому оптимальный подбор времени экспозиции ТЕМАН позволяет, с одной стороны, уменышить полное время процесса нанесения пленки, а с другой, снизить расход прекурсора.

При расчетах были использованы характерные для TEMAH параметры ALD-процесса [1], температура и давление в газовой фазе на шаге дозирования первого прекурсора равны соответственно $T_{g}=120^{\circ} \mathrm{C}$ и $P=1$ mTorr.

\section{1. Плотность потока частиц на боковую поверхность тренча из газовой фазы $\left(J_{e s}\right)$}

Пусть сечение тренча представляет собой равнобокую трапецию с углом наклона стенок $\alpha$, который считается положительным, если боковые стенки наклонены наружу (рис. 1, $a$ ). О площадку $\Delta S$ на боковой стенке тренча за время $\Delta t$ ударятся те частицы, направление скорости которых лежит в диапазоне углов $\theta \in\left(0 ; \theta_{\max }\right]$, где угол $\theta$ отсчитывается от стенки, на которую падают частицы. При этом связь между компонентами скорости частиц прекурсора и координатой $(z)$ площадки $\Delta S$ может быть получена из соотношения

$$
\operatorname{tg} \theta_{\max }=\frac{W \cos ^{2} \alpha}{z-W \sin \alpha \cos \alpha}=\frac{v_{\perp}^{\max }}{v_{\|}}
$$

где $v_{\|}, v_{\perp}-$ параллельная и перпендикулярная стенке компоненты скорости частиц.

C учетом максвелловского распределения частиц прекурсора по скоростям плотность их потока из газовой фазы на боковые стенки тренча будет

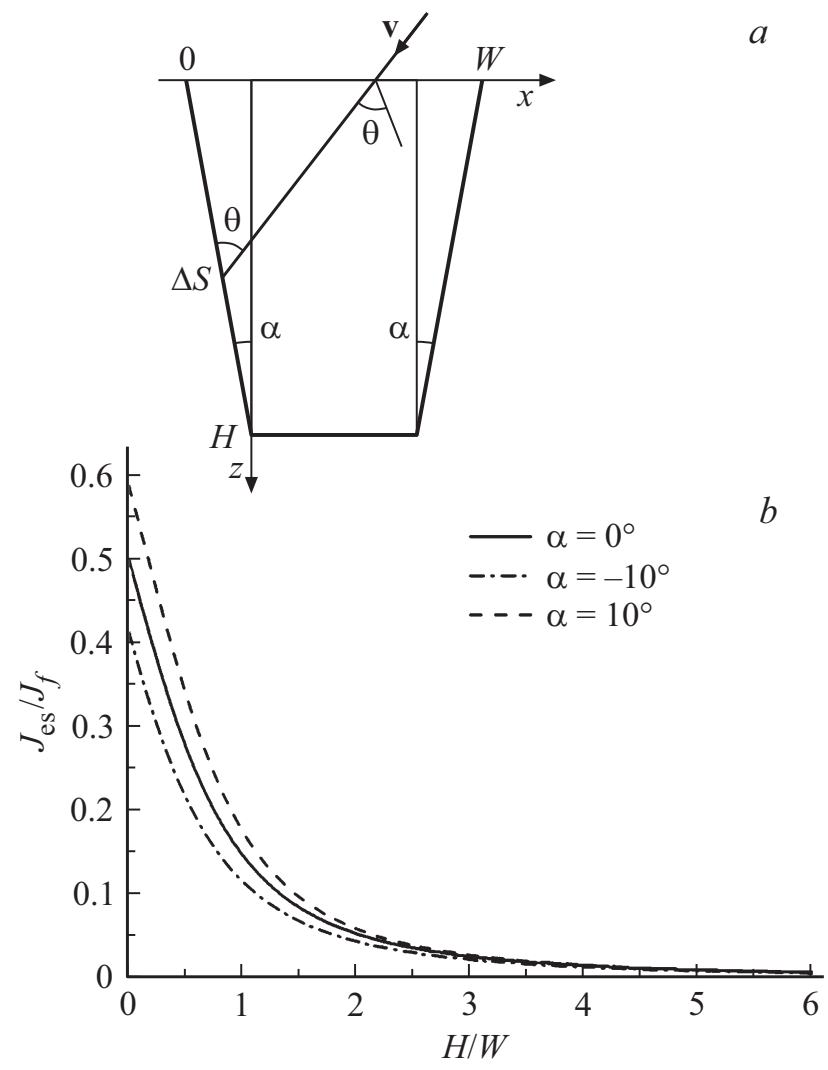

Рис. 1. Схема расчета плотности потока частиц из газовой фазы на боковую стенку тренча $(a)$, а также зависимость указанного потока от глубины при различных углах наклона боковых стенок тренча $(b)$.

определяться формулой:

$$
\begin{aligned}
J_{e s}=\left\{\begin{array}{l}
n_{g} \frac{m}{2 \pi k T_{g}} \int_{0}^{\infty} \exp \left(-\frac{m v_{\|}^{2}}{2 k T_{g}}\right) d v_{\|} \int_{0}^{v_{\perp}^{\max }} v_{\perp} \\
\times \exp \left(-\frac{m v_{\perp}^{2}}{2 k T_{g}}\right) d v_{\perp}, z-W \sin \alpha \cos \alpha \geq 0 \\
n_{g} \frac{m}{2 \pi k T_{g}} \int_{0}^{\infty} \exp \left(-\frac{m v_{\|}^{2}}{2 k T_{g}}\right) d v_{\|} \int_{v_{\perp}^{\max }}^{\infty} v_{\perp} \\
\times \exp \left(-\frac{m v_{\perp}^{2}}{2 k T_{g}}\right) d v_{\perp}, z-W \sin \alpha \cos \alpha<0
\end{array}\right\}= \\
\left.=\frac{J_{f}\left[1-\frac{1-2 \eta\left(W \sin ^{2} \cos \alpha-z\right)}{2}\right]}{\sqrt{1+\operatorname{tg}^{2} \theta_{\max }}}\right] \\
\approx|W \ll z| \approx \frac{J_{f}}{4}\left(\frac{W \cos ^{2} \alpha}{z}\right)^{2},
\end{aligned}
$$

где $\eta(z)$ - функция Хевисайда, а $J_{f}=n_{g} \sqrt{\frac{m}{2 \pi k T_{g}}} \times$ $\times \int_{0}^{\infty} v_{z} e^{-\frac{m v_{z}^{2}}{2 k T_{g}}} d v_{z}=n_{g} \sqrt{\frac{k T_{g}}{2 \pi m}}-$ плотность потока частиц 
массой $m$ на плоскую поверхность, $n_{g}$ и $T_{g}$ - концентрация и температура молекул прекурсора в газовой фазе над плоской поверхностью, $k$ - постоянная Больцмана.

На рис. $1, b$ показана зависимость плотности потока частиц ТЕМАН на стенку тренча из газовой фазы от глубины при различных углах наклона стенок тренча. При этом плотность потока частиц нормировалась на плотность потока, соответствующую плоской поверхности $\left(J_{f}\right)$, а координата - на ширину тренча $(W)$. Расчет проводился для тренча с исходным аспектным отношением $A=20$. Затенение потока частиц из газовой фазы над поверхностью на площадку $\Delta S$, расположенную на боковой стенке тренча, связано с величиной угла наклона стенок. Из рисунка видно, что сильнее всего наклон стенок тренча влияет на плотность потока частиц вблизи плоской поверхности и уменьшается по мере приближения ко дну тренча.

\section{2. Плотность потока частиц на дно тренча из газовой фазы $\left(J_{e b}\right)$}

Рассмотрим поток частиц на дно тренча из газовой фазы. О площадку $\Delta S$ на дне тренча за время $\Delta t$ (рис. 2) ударятся те частицы, направление скорости которых лежит в диапазоне углов $\left[\theta_{1} ; \theta_{2}\right]$, отсчитываемых от вертикали. Соотношение между компонентами скорости частиц прекурсора и положением $x$ площадки $\Delta S$ на дне тренча определяется выражениями

$$
\operatorname{tg} \theta_{1}=\frac{x}{H}=\frac{v_{x}^{\max 1}}{v_{z}}, \operatorname{tg} \theta_{2}=\frac{W-x}{H}=\frac{v_{x}^{\max 2}}{v_{z}} .
$$

В результате плотность потока частиц на дно тренча можно записать в виде

$$
\begin{aligned}
J_{e b} & =n_{g} \frac{m}{2 \pi k T_{g}} \int_{0}^{\infty} v_{z} \exp \left(-\frac{m v_{z}^{2}}{2 k T_{g}}\right) d v_{z} \\
& \times \int_{-v_{x}^{\max } 1}^{v_{x}^{\max 2}} \exp \left(-\frac{m v_{x}^{2}}{2 k T_{g}}\right) d v_{x} \\
& =\frac{J_{f}}{2}\left(\frac{x}{\sqrt{x^{2}+H^{2}}}+\frac{W-x}{\sqrt{(W-x)^{2}+H^{2}}}\right) \\
& \approx|W \ll z| \approx \frac{J_{f}}{2} \frac{W}{H} .
\end{aligned}
$$

Полученная формула не содержит $\alpha$. Влияние наклона стенок на поток у дна проявляется в том, что координата $x$ на дне тренча изменяется в пределах от $H \operatorname{tg} \alpha$ на левой границе до $W-H \operatorname{tg} \alpha$ на правой границе. Плотность внешнего потока частиц прекурсора на дно тренча спадает по мере удаления от его центра (6). Внутри области $[0 ; W]$ поток частиц не зависит от угла наклона стенок, что следует из формулы (6). При больших аспектных отношениях $(A=20)$ неоднородность внешнего потока частиц на дно тренча составляет менее $0.5 \%$.

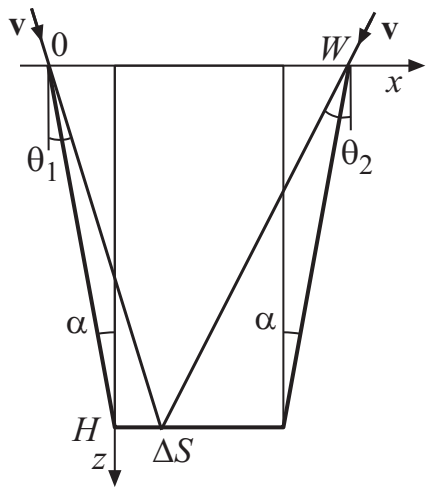

Рис. 2. Схема расчета плотности потока частиц из газовой фазы на дно тренча.

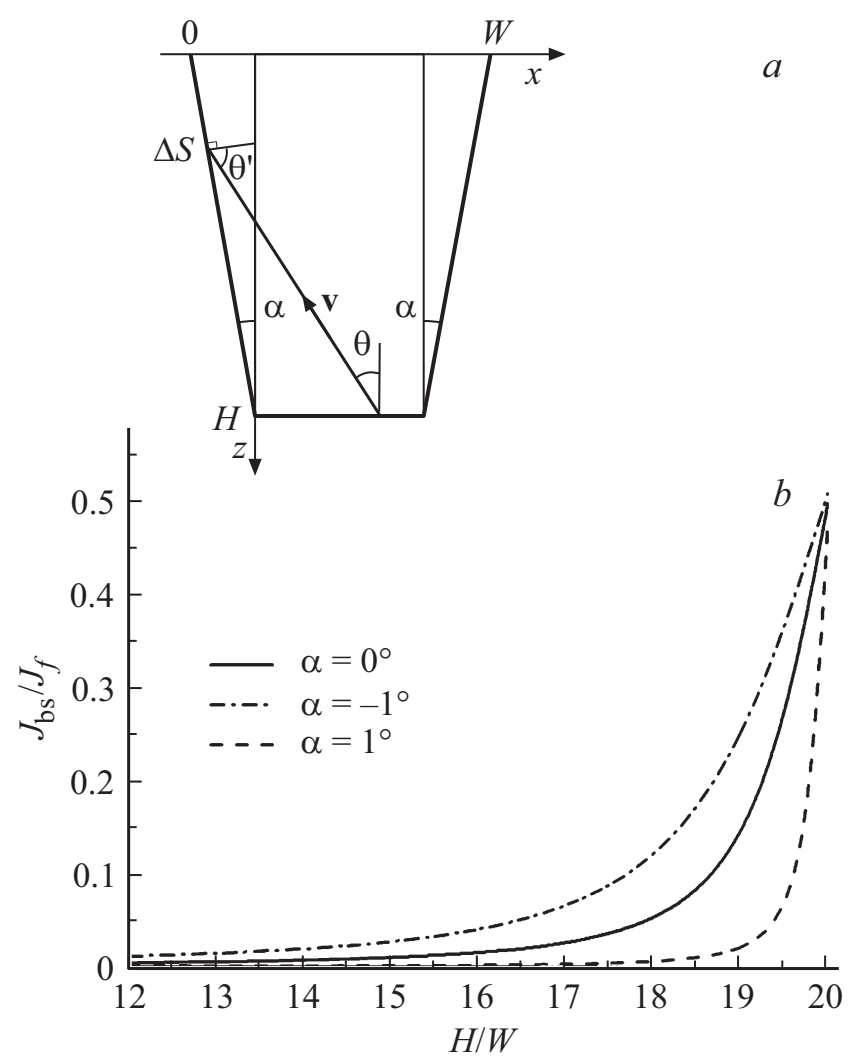

Рис. 3. Схема расчета плотности потока частиц на боковую стенку тренча за счет частиц, отраженных от его дна $(a)$, а также зависимость этого потока от глубины при различных углах наклона боковых стенок тренча $(b)$.

\section{3. Плотность потока частиц на стенки тренча, индуцированная отражением от дна $\left(J_{b s}\right)$}

Обозначим за $J_{b}(x)$ плотность потока частиц на дно тренча, который определяется суммой внешнего потока $\left(J_{e b}\right)$ и потока частиц, отраженных от боковых стенок тренча $\left(J_{s b}\right)$. Внутрь телесного угла $\Delta \Omega$ по направлению к площадке $\Delta S$ на боковой стенке тренча (рис. 3,a), согласно закону косинуса, полетит 
$(1-s(1-\Theta(x, H))) J_{b}(x) d x d y \frac{\Delta \Omega}{\pi} \cos \theta$ частиц, где

$$
\begin{aligned}
& \Delta \Omega=\frac{\Delta S \cos \theta^{\prime}}{(H-z)^{2}+(x-z \operatorname{tg} \alpha)^{2}+y^{2}}, \\
& \cos \theta=\frac{H-z}{\sqrt{(H-z)^{2}+(x-z \operatorname{tg} \alpha)^{2}+y^{2}}}, \\
& \cos \theta^{\prime}=\frac{(x-H \operatorname{tg} \alpha) \cos \alpha}{\sqrt{(H-z)^{2}+(x-z \operatorname{tg} \alpha)^{2}+y^{2}}} .
\end{aligned}
$$

Интегрируя по площади дна, получим выражение для плотности потока частиц на площадку $\Delta S$ боковой стенки тренча за счет частиц, отраженных от его дна:

$$
\begin{aligned}
J_{b s}(z) & =\int_{H \operatorname{tg} \alpha}^{W-H \operatorname{tg} \alpha}(1-s(1-\Theta(x, H))) J_{b}(x) d x \\
& \times \int_{-\infty}^{\infty} d y \frac{\cos \theta^{\prime}}{(H-z)^{2}+(x-z \operatorname{tg} \alpha)^{2}+y^{2}} \frac{1}{\pi} \cos \theta \\
& =\frac{(H-z) \cos \alpha}{2} \int_{H \operatorname{tg} \alpha}^{W-H \operatorname{tg} \alpha}(1-s(1-\Theta(x, H))) \\
& \times\left(J_{e b}(x) \sqrt{\frac{T_{x}}{T_{g}}}+J_{s b}(x)\right) \\
& \times \frac{x-H \operatorname{tg} \alpha}{\left((H-z)^{2}+(x-z \operatorname{tg} \alpha)^{2}\right)^{3 / 2}} d x .
\end{aligned}
$$

Множитель $\sqrt{T_{s} / T_{g}}$ в (8) учитывает, что молекулы после удара о поверхность приобретают температуру подложки.

На рис. $3, b$ изображена зависимость отраженного от дна тренча потока на его стенки как функция глубины. График получен для случая, когда нормированная плотность падающего потока на дно тренча с аспектным отношением $A=20$ всюду равна единице, т. е. $(1-s(1-\Theta(x, H)))\left(J_{e b}(x) \sqrt{\frac{T_{s}}{T_{g}}}+J_{s b}(x)\right)=J_{f}$. Из рисунка видно, что полученные кривые значительно отличаются даже при малых углах наклона стенок тренча от вертикали $\left(\alpha= \pm 1^{\circ}\right)$. Рост потока при уменьшении угла наклона стенок тренча обусловлен ростом площади поверхности дна тренча и, как следствие, потоком отраженных от дна частиц. Наибольшие отличия кривых наблюдаются вблизи дна тренча, из-за затухания величины отраженного потока по мере удаления от его дна.

Оптимальное время подачи прекурсора $\left(t_{c}-\right.$ критическое время) определяется минимальным временем, необходимым для монослойного покрытия стенок тренча. Поскольку область, отвечающая минимальному потоку частиц, находится вблизи дна тренча, то наклон его стенок может приводить к заметному изменению критического времени подачи прекурсора.

\section{4. Плотность потока частиц на дно тренча, индуцированная отражением от его стенок $\left(J_{s b}\right)$}

Рассмотрим поток частиц на дно тренча за счет отражения от его стенок. Число молекул, ударившихся в единицу времени об участок поверхности $\Delta S=d y d z / \cos \alpha$ боковой стенки тренча (рис. 4), равно $J_{s}(z) d y d z / \cos \alpha$, где $J_{s}(z)$ - плотность потока частиц на боковую стенку тренча. Данный поток определяется суммой внешнего потока $\left(J_{e s}\right)$ и потоков, отраженного от дна $\left(J_{b s}\right)$ и от противоположной стенки $\left(J_{s s}\right)$ тренча. Среди ударившихся о боковую стенку частиц внутри телесного угла $\Delta \Omega$ по направлению к площадке на дне тренча, согласно закону косинуса, направится число частиц $p(0, z) J_{a}(z) \frac{d y d z}{\cos \alpha} \frac{\Delta \Omega}{\pi} \cos \theta^{\prime}$.

Принимая во внимание выражение для телесного угла $\Delta \Omega=\frac{\Delta S \cos \theta}{(H-z)^{2}+(x-z)^{2}+(x-z \operatorname{tg} \alpha)^{2}+y^{2}}$, после интегрирования по $y$ и глубине тренча получим плотность потока частиц прекурсора, отраженных от левой боковой стенки тренча, на его дно

$$
\begin{aligned}
\tilde{J}_{s b}(x) & =\frac{x-H \operatorname{tg} \alpha}{\pi} \int_{0}^{H}\left(1-s\left(1-\Theta_{s}(z)\right)\right) J_{s}(z)(H-z) d z \\
& \times \int_{-\infty}^{\infty} \frac{d y}{\left((H-z)^{2}+(x-z)^{2}+y^{2}\right)^{2}} \\
& =\frac{x-H \operatorname{tg} \alpha}{2} \int_{0}^{H}\left(1-s\left(1-\Theta_{s}(z)\right)\right) J_{s}(z) \\
& \times \frac{(H-z)}{\left((H-z)^{2}+(x-z \operatorname{tg} \alpha)^{2}\right)^{3 / 2}} d z .
\end{aligned}
$$

Поток, отраженный от правой боковой стенки тренча, будет иметь тот же вид с заменой $x$ на $W-x$. Полный отраженный поток на дно тренча будет равен сумме потоков от каждой из стенок независимо. Из симметрии задачи следует, что степень покрытия $\Theta_{s}(z)$ и плотность потока частиц $J_{s}(z)$ имеют одинаковые значения для

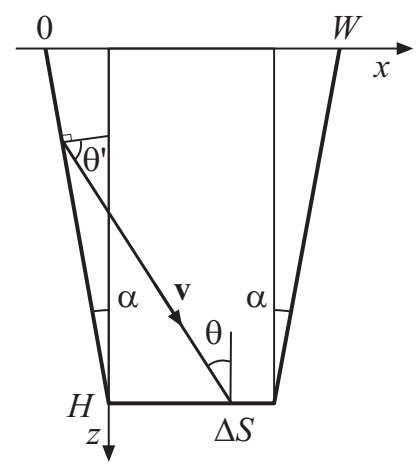

Рис. 4. Схема расчета плотности потока частиц на дно тренча за счет частиц, отраженных от его боковых стенок. 


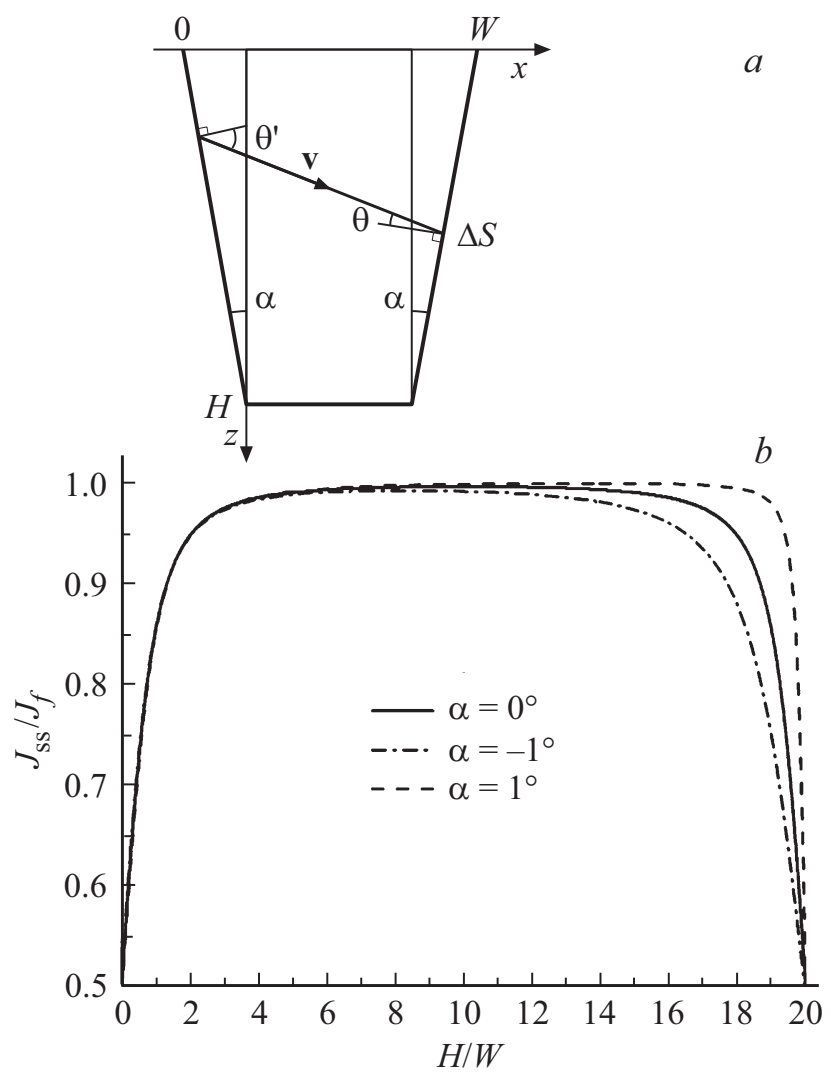

Рис. 5. Схема расчета плотности потока частиц на боковую стенку тренча за счет частиц, отраженных от его противоположенной стенки $(a)$, а также зависимость этого потока от глубины при различных углах наклона боковых стенок тренча $(b)$.

левой и правой стенок тренча. В результате выражение для $J_{s b}(x)$ примет вид

$$
\begin{aligned}
& J_{s b}(x)=\frac{1}{2} \int_{0}^{H}\left(1-s\left(1-\Theta_{s}(z)\right)\right) \\
& \times\left(J_{e s}(z) \sqrt{\frac{T_{s}}{T_{g}}}+J_{b s}(z)+J_{s s}(z)\right) \\
& \times\left[\frac{x-H \operatorname{tg} \alpha}{\left((H-z)^{2}+(x-z \operatorname{tg} \alpha)^{2}\right)^{3 / 2}}\right. \\
& \left.+\frac{W-z-H \operatorname{tg} \alpha}{\left((H-z)^{2}+(W-x-z \operatorname{tg} \alpha)^{2}\right)^{3 / 2}}\right](H-z) d z .
\end{aligned}
$$

Как и в случае внешнего потока $\left(J_{e s}\right)$, наклон стенок тренча не вносит существенного влияния в поток на дно тренча, обусловленный отражением от его стенок. При условии

$$
\left(1-s\left(1-\Theta_{s}(z)\right)\right)\left(J_{e s}(z) \sqrt{\frac{T_{s}}{T_{g}}}+J_{b s}(z)+J_{s s}(z)\right)=J_{f}
$$

поток на дно тренча является однородным и не зависящим от угла наклона его боковых стенок.

\section{5. Плотность потока частиц на боковую стенку тренча, индуцированная отражением от противоположенной стенки $\left(J_{s s}\right)$}

Среди частиц, ударившихся об элемент поверхности $d y d z_{z} / \cos \alpha$ боковой стенки тренча, внутрь телесного угла $\Delta \Omega$ по направлению к площадке $\Delta S$ (рис. $5, a)$, находящейся на противоположенной стенке и имеющей координату $z$, полетит $p\left(0, z_{1}\right) J_{s}\left(z_{1}\right) \frac{d y d z_{1}}{\cos \alpha} \frac{\Delta \Omega}{\pi} \cos \theta^{\prime}$ частиц. Поток $J_{s}$ обусловлен суммой внешнего потока $\left(J_{e s}\right)$, потока, отраженного от дна тренча $\left(J_{b s}\right)$, и потока, отраженного от противоположенной стенки $\left(J_{s s}\right)$ тренча.

Учитывая, что

$$
\begin{gathered}
\Delta \Omega=\frac{\Delta S \cos \theta}{\left.\left(W-\left(z+z_{1}\right) \operatorname{tg} \alpha\right)\right)^{2}+y^{2}+\left(z-z_{1}\right)^{2}}, \\
\cos \theta=\frac{W-2 z \operatorname{tg} \alpha}{\sqrt{\left(W-\left(z+z_{1}\right) \operatorname{tg} \alpha\right)^{2}+y^{2}+\left(z-z_{1}\right)^{2}}} \cos \alpha, \\
\cos \theta^{\prime}=\frac{W-2 z_{1} \operatorname{tg} \alpha}{\sqrt{\left(W-\left(z+z_{1}\right) \operatorname{tg} \alpha\right)^{2}+y^{2}+\left(z-z_{1}\right)^{2}}} \cos \alpha,
\end{gathered}
$$

после интегрирования по $d z_{1}$ получим выражение для плотности потока частиц, отраженных от одной из боковых стенок и попавших на противоположенную стенку на расстоянии $z$ от поверхности:

$$
\begin{aligned}
& J_{s s}(z)=\frac{\cos \alpha}{\pi} \int_{0}^{H}\left(1-s\left(1-\Theta\left(0, z_{1}\right)\right)\right) J_{s}\left(z_{1}\right) d z_{1} \\
& \times \int_{-\infty}^{\infty} \frac{\left(W-2 z_{1} \operatorname{tg} \alpha\right)(W-2 z \operatorname{tg} \alpha)}{\left(\left(W-\left(z+z_{1}\right) \operatorname{tg} \alpha\right)^{2}+y^{2}+\left(z-z_{1}\right)^{2}\right)^{2}} d y \\
& =\frac{\cos \alpha}{2} \int_{0}^{H}\left(1-s\left(1-\Theta\left(0, z_{1}\right)\right)\right) \\
& \times\left(J_{e s}\left(z_{1}\right) \sqrt{\left.\frac{T_{s}}{T_{g}}+J_{b s}\left(z_{1}\right)+J_{s s}\left(z_{1}\right)\right)}\right. \\
& \times \frac{\left(W-2 z_{1} \operatorname{tg} \alpha\right)(W-2 z \operatorname{tg} \alpha)}{\left(\left(W-\left(z+z_{1}\right) \operatorname{tg} \alpha\right)^{2}+\left(z-z_{1}\right)^{2}\right)^{3 / 2}} d z_{1} .
\end{aligned}
$$

Зависимость потока на боковую стенку тренча за счет отражения от его противоположенной стенки как функция глубины показана на рис. 5, $b$ при условии

$$
\begin{aligned}
& \left(1-s\left(1-\Theta\left(0, z_{1}\right)\right)\right) \\
& \times\left(J_{e s}\left(z_{1}\right) \sqrt{\frac{T_{s}}{T_{g}}}+J_{b s}\left(z_{1}\right)+J_{s s}\left(z_{1}\right)\right)=J_{f} .
\end{aligned}
$$


Как можно видеть, существенное изменение отраженного от боковых стенок тренча потока с изменением наклона его боковых стенок проявляется в области вблизи дна тренча. Вблизи поверхности тренча кривые, соответствующие разным углам наклона, фактически совпадают. Полученный результат обусловлен тем, что с уменьшением угла наклона стенок тренча все большее число отраженных от боковой стенки частиц попадает на дно тренча, а не на его противоположенную стенку.

При оптимизации времени подачи прекурсора для конформного покрытия стенок тренча необходимо подобрать минимальное время, при котором наиболее труднодоступная для прекурсора область на стенках тренча вблизи дна будет иметь монослойное покрытие. Исследование зависимости потоков от угла наклона стенок тренча показала, что наибольшее влияние наклон боковых стенок тренча в данной области оказывает на отраженные потоки $J_{b s}$ и $J_{s s}$. Поэтому ими в первую очередь будут определяться критическое время подачи прекурсора и профиль растущей пленки.

\section{2. Профиль толщины пленки при варьировании угла наклона стенок тренча}

Используя выражения для плотностей потоков частиц на стенки (4), (8), (12) и дно (6), (10) тренча, запишем систему интегро-дифференциальных уравнений:

$$
\left\{\begin{array}{l}
n_{0} \frac{d \Theta(0, z)}{d t}=\left(J_{e s}(z)+J_{s s}(z)+J_{b s}(z)\right)(s(1-\Theta(0, z)), \\
n_{0} \frac{d \Theta(0, H)}{d t}=\left(J_{e b}(x)+J_{s b}(x)\right) s(1-\Theta(x, H)) .
\end{array}\right.
$$

Система (13) решалась численно для различных значений коэффициента прилипания $(s)$, угла наклона стенок $(\alpha)$ и времени подачи первого прекурсора TЕМАН $(t)$. Время подачи второго прекурсора (воды) полагалось много большим критического значения $\left(t \gg t_{c}\right)$. Поэтому профиль пленки полностью определялся временем подачи первого прекурсора. Для определенности в расчетах предполагалось, что коэффициент прилипания молекул ТЕМАН равен $s=0.5$.

Исследуем, как меняется конформность покрытия поверхности тренча в зависимости от угла наклона его боковых стенок. Для этого при решении системы (13) ширина и глубина тренча выбирались равными $W=160 \mathrm{~nm}$ и $H=4 \mu \mathrm{m}$, что соответствует аспектному отношению $A=25$. Следует заметить, что при угле наклона боковых стенок тренча $\alpha=+1^{\circ}$ ширина дна тренча будет составлять менее $13 \%$ от ширины входного отверстия. Время подачи ТЕМАН было выбрано меньше критического и составляло $t=0.2 \mathrm{~s}$. На рис. 6 наблюдается уменьшение степени покрытия поверхности тренча с ростом угла наклона его боковых стенок. Данный результат связан с тем, что с увеличением угла наклона боковых стенок растет число частиц, покидающих тренч
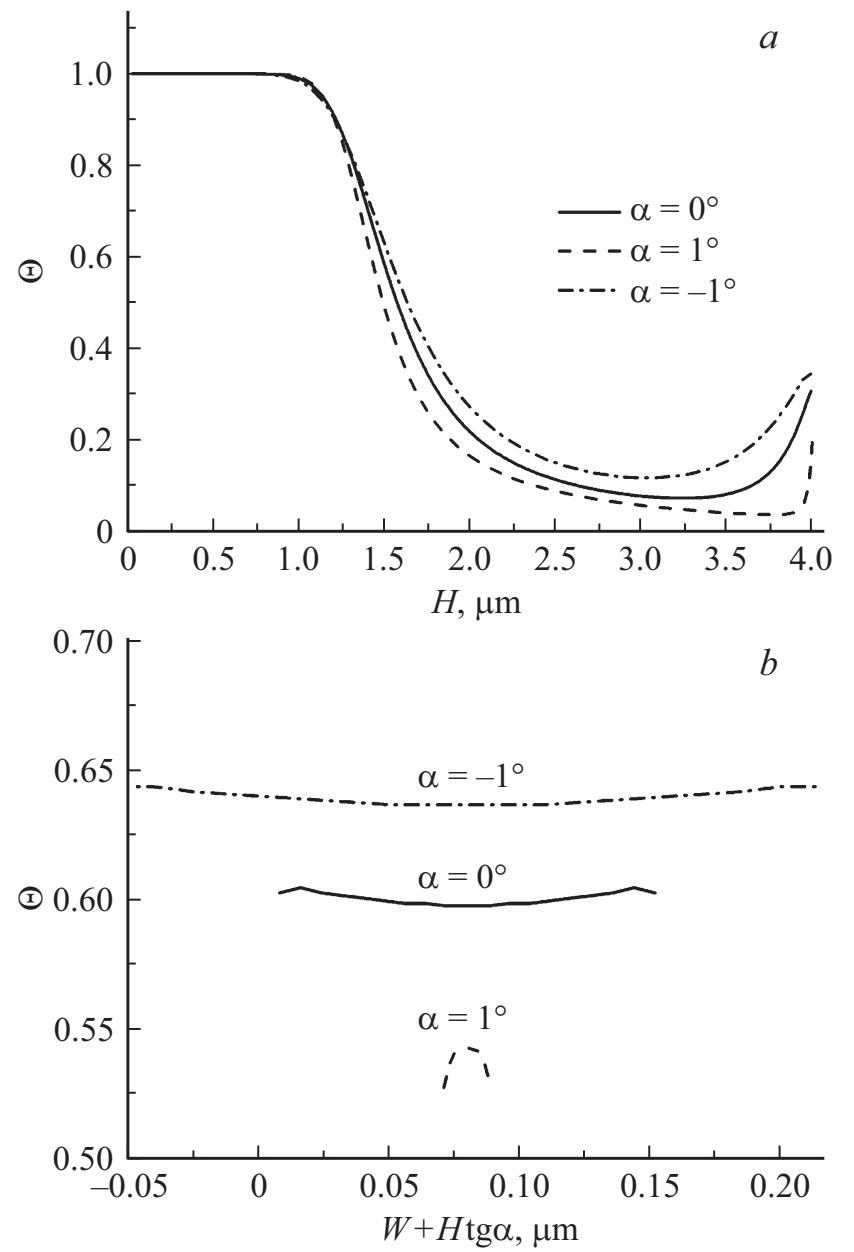

Рис. 6. Степень покрытия боковой стенки тренча от глубины $(a)$ и степень покрытия вдоль дна тренча от координаты $(b)$ при различных углах наклона боковых стенок $(A=25)$.

после отражения от его боковых стенок. На рис. 6, $a$ можно наблюдать смещение минимума степени заполнения стенок тренча в область больших глубин. Указанное смещение происходит из-за уменьшения плотности потока $\left(J_{b s}\right)$ отраженных от дна тренча частиц прекурсора на боковые стенки. Уменьшение плотности отраженного потока от дна тренча вызвано как уменьшением площади дна при наклонных стенках, так и уменьшением угла падения отраженных частиц на боковые стенки. Критическое время подачи прекурсора, как было показано в [8], лимитируется временем конформного покрытия его боковых стенок. Положим, что достаточная степень конформности составляет 95\%. Расчеты, согласно (13), показывают, что эффект уменьшения плотности потока частиц на стенки тренча с ростом угла их наклона приводит к росту критического времени подачи прекурсора (рис. 7). Как можно видеть из рисунка, влияние отклонения боковых стенок от вертикали на критическое время подачи прекурсора резко возрастает с ростом аспектного отношения. Крайние точки кривых при $\alpha>0$ на рис. 7 соответствуют случаю, когда ширина дна составляет 
менее $10 \%$ от ширины входного отверстия. Резкий рост $t_{c}$ при положительных углах наклона стенок тренча обусловлен смещением минимума степени заполнения поверхности боковых стенок тренча в область больших глубин, поскольку плотность потока частиц на боковые стенки быстро падает с глубиной. Очевидно, что при временах подачи прекурсора, меньших критического, динамический угол наклона стенок тренча по мере увеличения количества ALD-циклов будет только расти, что в свою очередь приведет к снижению конформности осаждаемой пленки.

Далее исследуем, исходя из (13), как изменение динамического аспектного отношения тренча по мере роста пленки сказывается на профиле ее толщины. Для этого исходная ширина и глубина были выбраны равными $(W=60 \mathrm{~nm}$ и $H=2 \mu \mathrm{m})$, что соответствует аспектному отношению $A=33$. При данной ширине тренча изменение аспектного отношение от начального значения составит $40 \%$ после 120 ALD-циклов (за 1 цикл выращивается $0.1 \mathrm{~nm}$ пленки). На рис. 8 представлена зависимость приведенной к количеству ALD-циклов степени заполнения боковой стенки тренча после 1, 60 и 120 ALD-циклов. Для выделения эффекта, обусловленного исключительно динамическим изменением аспектного отношения по мере роста пленки, начальный угол наклона стенок тренча был выбран равным $\alpha=0^{\circ}$ и неизменным в процессе проведения ALD-циклов. Время подачи прекурсора (ТЕМАН) задавалось равным $t=0.2 \mathrm{~s}$. При указанных условиях проведение каждого последующего ALD-цикла приводит к увеличению аспектного отношения тренча. И, как следствие, к уменьшению глубины конформного покрытия стенок тренча (рис. 8), а также к необходимости увеличения критического времени подачи прекурсора [8].

Изменение аспектного отношения уменьшается монотонно с ростом числа ALD-циклов. Поэтому можно предположить, что аспектное отношение, соответству-

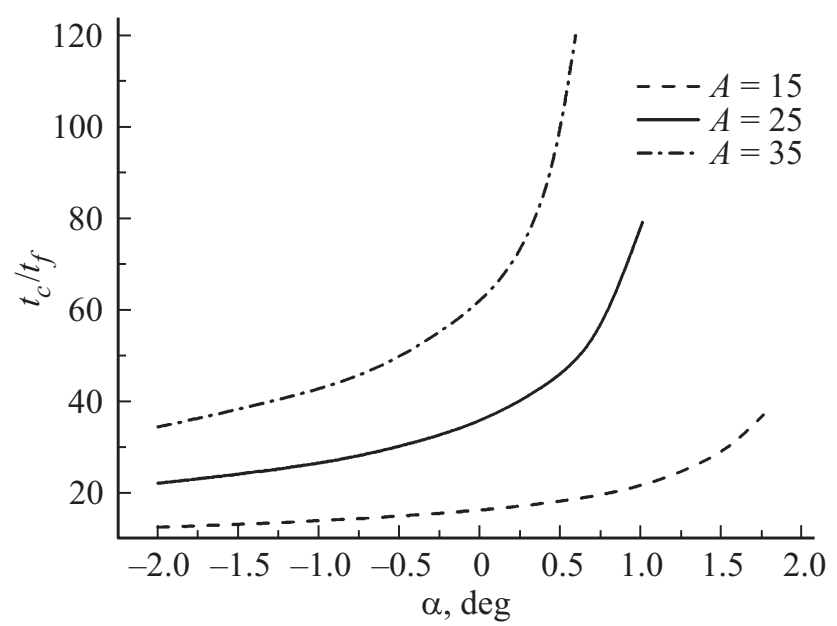

Рис. 7. Зависимость критического времени подачи прекурсора от угла наклона боковых стенок тренча для различных аспектных отношений.

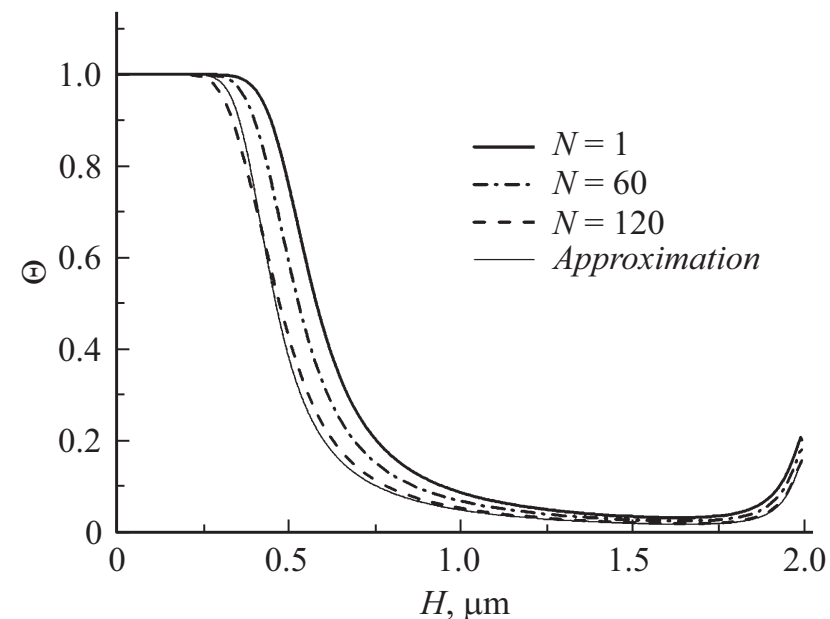

Рис. 8. Приведенная к числу ALD-циклов степень покрытия боковой стенки тренча от глубины для различного числа ALD-циклов.

ющее половине от необходимого числа циклов, будет являться аппроксимацией реального профиля выращенной пленки. На рис. 8 изображена аппроксимационная кривая для профиля, соответствующего 120 ALD-циклам. Полученный профиль будет иметь пленка после одного ALD-цикла в тренче с аспектным отношением $A=42$, соответствующим половине от необходимого числа циклов $(N=60)$. Данная кривая описывает профиль растущей пленки со среднеквадратичным отклонением менее $10 \%$ и может использоваться как начальное приближение при определении критического времени подачи прекурсора и верификации экспериментальных данных.

\section{Заключение}

В настоящей работе проведено теоретическое исследование влияния исходного наклона стенок тренча, его возможного изменения, а также увеличения аспектного отношения по мере роста пленки на однородность толщины (конформность) пленок, осаждаемых на поверхность тренчей методом ALD.

Аналитическая модель основана на использовании кинетических уравнений, описывающих степень заполнения элемента поверхности тренча в зависимости от времени подачи прекурсора. Получены аналитические выражения для плотностей потоков частиц на стенки и дно тренча с учетом отклонения боковых стенок от вертикали.

Проведено исследование зависимости степени заполнения поверхности тренча при заданном времени подачи прекурсора от угла наклона боковых стенок. Показано, что отклонение угла наклона стенок высокоаспектного тренча от вертикали приводит к уменьшению конформности растущей пленки. С увеличением положительных углов наклона боковых стенок тренча происходит 
резкий рост критического времени подачи прекурсора. Причем изменения усиливаются с ростом количества ALD-циклов, если дозирование прекурсора изначально недостаточно для конформного покрытия стенок тренча. Этот эффект при отрицательных углах наклона стенок выражен гораздо слабее.

Для наноразмерных тренчей была исследована зависимость профиля толщины растущей пленки от количества проведенных ALD-циклов. Получено, что изменение acпектного отношения по мере роста пленки необходимо учитывать, когда ширина тренча уменьшается более чем на 10\% после выращивания пленки необходимой толщины.

Таким образом, результаты моделирования показали значительное влияние начального угла наклона боковых стенок тренча на конформность получаемой пленки, а также необходимость учета динамического изменения угла наклона стенок тренча и аспектного отношения с ростом количества ALD-циклов.

\section{Список литературы}

[1] Atomic Layer Deposition of Nanostructured Materials / Eds. by N. Pinna, M. Knez. Wiley-VCH Verlag \& Co. KGaA, 2012. $435 \mathrm{p}$.

[2] Wulu C., Saraswat R.C., McVittie J.P. // J. Electrochem. Soc. 1991. Vol. 138. N 6. P. 1831-1840.

[3] Elam J.W., Routkevitch D., Mardilovich P.P., George S.M. // Chem. Mater. 2003. Vol. 15. P. 3507-3517.

[4] Rose M., Bartha J.W. // Appl. Surf. Sci. 2009. Vol. 255. P. 6620-6623.

[5] Knoops H.C.M., Langereis E., van de Sanden M.C.M., Kessels M.M. // J. Electrochem. Soc. 2010. Vol. 57. N 12. P. G241-G249.

[6] Yanguas-Gil A., Elam J.W. // ECS Trans. 2011. Vol. 41. N 2. P. 169-174.

[7] Yanguas-Gil A., Elam J.W. // Chem. Vap. Depos. 2012. Vol. 18. P. 46-52.

[8] Фадеев А.В., Мяконьких А.В., Руденко К.В. // ЖТФ. 2018. Т. 88. Вып. 2. С. 243-250. 